Substitution of equation (20) into equation (15) gives a condition for instability:

$$
\frac{4}{(\sqrt{\pi) D(\bar{c})}} \cdot \frac{\mathrm{d} D}{\mathrm{~d} \bar{c}} \cdot \bar{c}<\operatorname{Real}(q)<0 .
$$

It is evident that the more negative the term $\mathrm{d} D / \mathrm{d} \bar{c}$ is the larger the domain of $q$ where instabilities occur. In the case of a constant diffusion coefficient, inequalities (20) and (21) reveal that no instability can occur.

The above analysis does not confirm the hypothesis that a minimum in the $D$ c c relationship is necessary to induce instability but since such a configuration will contain a negative $\mathrm{d} D / \mathrm{d} c$ slope, such systems will give instabilities for concentrations up to the minimum value. Furthermore the analysis explains why some systems exhibit instability under conditions of absorption but not desorption and vice-versa.

\section{REFERENCES}

1. J. C. Berg, Recent Developments in Separation Science, Vol. 2, pp. 1-31. Chemical Rubber Co., Cleveland, $\mathrm{OH}$ (1972).

2. K. C. Pratt and W. A. Wakeham. The mutual diffusion coefficient of ethanol-water mixtures: determination by a rapid, new method, Proc. $R$. Soc. A336, 393-406 (1974).

3. M. T. Tyn and W. F. Calus, Temperature and concentration dependence of mutual diffusion coefficients of some binary liquid systems, $J$. Chem. Engng Data 20. $310-316$ (1975).

4. H. Ertl, R. K. Ghai and F. A. L. Dullien, Liquid diffusion of nonelectrolytes, A.I.Ch.E. Jl 19. $881-900$ (1973): Supplement Document No. 02172, NAPS, New York $(1973)$

5. R. B. Bird, W. E. Stewart and E. N. Lightfoot, Transpon Phenomena, p. 537. John Wiley, New York (1960).

\title{
THE DISTURBANCE OF A UNIFORM STEADY-STATE HEAT FLUX BY A PARTIALLY CONDUCTING PLANE CRACK
}

\author{
J. R. BARBER
}

University Department of Mechanical Engineering, Newcastle IJpon Tyne, NEI 7RU, England

(Received 12 November 1975 and in revised form 6 February 1976)

\section{NOMENCLATURE}

$x, y, z$, cartesian co-ordinates;

$r, \theta, z, \quad$ cylindrical polar co-ordinates;

$\rho, \zeta, \quad$ non-dimensional polar co-ordinates;

$A, \bar{A}$, extent of the crack and remainder of the crack plane respectively:

a. crack radius or semi-width:

$\delta_{i j}$, Kronecker delta;

$h$, thermal contact resistance:

$j(t)$, odd function of $t$ defined by equation (8);

$K$, thermal conductivity;

$\phi$. $\quad \arccos t$

4: heat flux density in $z$ direction:

$s, t$ parameters of integration :

$T$, temperature.

\section{INTRODUCTION}

IF A THERMALLY conducting solid contains a small plane crack, the temperature field in the vicinity of the crack will be perturbed from that in an otherwise similar unflawed solid. The extreme case of a completely insulated crack leads to a classical mixed boundary value problem in potential theory, a solution for the penny-shaped crack being given by Karush and Young [1]. However, a more realistic boundary condition is that of "radiation" across the crack, proportional to the local discontinuity in temperature. This leads to a mixed boundary value problem of the third type which is here solved for the penny-shaped crack and the "Griffith" crack, using a technique developed in another context [2].

The same solution applics to the problem of a cooled semi-infinite solid, part of whose surface is obstructed.

It is hoped subsequently to use these results to find the thermal stresses in a solid containing a partially conducting crack.

\section{STATEMENT OF THE PROBLEM}

We define a system of cartesian co-ordinates $(x, y, z)$ and polar co-ordinates $(r, \theta, z)$ such that the crack lies in the plane $z=0$, denoting the extent of the crack by $A$ and the rest of this plane by $\bar{A}$.

We assume that there is a uniform heat flux, $q_{0}$. in the $z$ direction at the extremities of the solid, i.e.

$$
q_{z}=-K \frac{i T}{\hat{\imath} z} \rightarrow q_{0}, \quad z \rightarrow \pm x .
$$

where $T$ is the temperature and $K$ the conductivity of the material.

In view of the antisymmetry of the problem there is no loss in generality in taking

$$
T=0, \text { on } \bar{A} \text {, }
$$

in which case the local temperatures on opposite sides of the crack must be equal and opposite.

If the heat flux through the crack is proportional to the local temperature difference across it, we have

$$
y_{z}=-2 h T \text { on } A, \quad z=0^{+} .
$$

where $h$ is the constant of proportionality.

Denoting the perturbation in temperature field due to the crack by $T_{1}$, such that

$$
T=T_{1}-\frac{40 z}{K}
$$

and substituting for $T$ into equation (1)-(3), we find

$$
\begin{gathered}
\frac{\partial T_{1}}{\hat{\imath} z} \rightarrow 0 . \quad z \rightarrow \infty . \\
T_{1}=0, \text { on } \bar{A} . \\
\frac{\partial T_{1}}{\partial z}-\frac{2 h T_{1}}{K}=\frac{q_{0}}{K}, \text { on } A, z=0^{+} .
\end{gathered}
$$


The problem is therefore to find a harmonic potential function, $T_{1}$, in the half-space $z>0$, satisfying the boundary conditions (5)-(7). These boundary conditions are also met in certain thermoelastic contact problems (cf. equation $(13 \mathrm{~b}, \mathrm{c})$ of [2] and we can therefore make use of existing solutions for the cases where $A \equiv|r|<a$ and $|x|<a$, corresponding to the penny-shaped crack of radius $a$ and the Griffith crack of length $2 a$ respectively.

\section{THE PENNY-SHAPED CRACK: $A \equiv|\boldsymbol{r}|<\boldsymbol{a}$}

Following the same procedure as in [2], we can satisfy equations $(5,6)$ by representing the distribution of temperature $T_{1}(r, z)$ in $z>0$ as

$$
T_{1}(r, z)=\frac{1}{2 \mathbf{i}} \int_{-1}^{+1} \frac{j(t) \mathrm{d} t}{\left[\rho^{2}+(\zeta+\mathbf{i} t)^{2}\right]^{\frac{1}{2}}},
$$

where $\rho=r / a ; \zeta=z / a$ and $j(t)$ is an odd function of $t$ to be determined from equation (7). This method of representation is developed and discussed by Collins [3] who shows that $j(t)$ must satisfy the Fredholm integral equation

$$
j(t)-\frac{2 h a}{\pi K} \int_{-1}^{+1} j(s) \log |s-t| \mathrm{d} s=\frac{2 q_{0} a t}{\pi K} .
$$

As $\zeta \rightarrow 0$, the values of the integrand in equation (8) at $t,-t$ respectively become equal if $t>\rho$ and self-cancelling if $\rho>t$. Hence, on the crack surface,

$$
T_{1}\left(r, 0^{+}\right)=-\int_{\rho}^{1} \frac{j(t) \mathrm{d} t}{\sqrt{\left(t^{2}-\rho^{2}\right)}}, \quad 0 \leqslant \rho \leqslant 1 .
$$

A convenient numerical solution can be obtained by writing $t=\cos \phi$ and representing $j(\phi)$ in the form

$$
j(\phi)=\sum_{i=1}^{\infty} a_{i} \cos (2 i-1) \phi, \quad 0 \leqslant \phi \leqslant \pi,
$$

in which case the integral in equation (10) can be evaluated [2] to give the set of equations

$$
\frac{2 h a}{K} \sum_{j=1}^{\infty} b_{i i} a_{j}+(2 i-1) a_{i}=\frac{2 q_{0} a \delta_{i 1}}{\pi K}, \text { for } i=1,2,3 \ldots
$$

for the coefficients $a_{i}$, where

$$
b_{i j}=-\frac{2}{\pi}\left\{\frac{1}{4(i-j)^{2}-1}+\frac{1}{4(i+j-1)^{2}-1}\right\}
$$

and $\delta_{i j}$ is the Kronecker delta.

Figure 1 shows the form of the temperature profile across the crack for various values of the non-dimensional parameter $2 h a / K$. Thesc results were obtained by truncating the series for $j(\phi)$ to twelve terms.

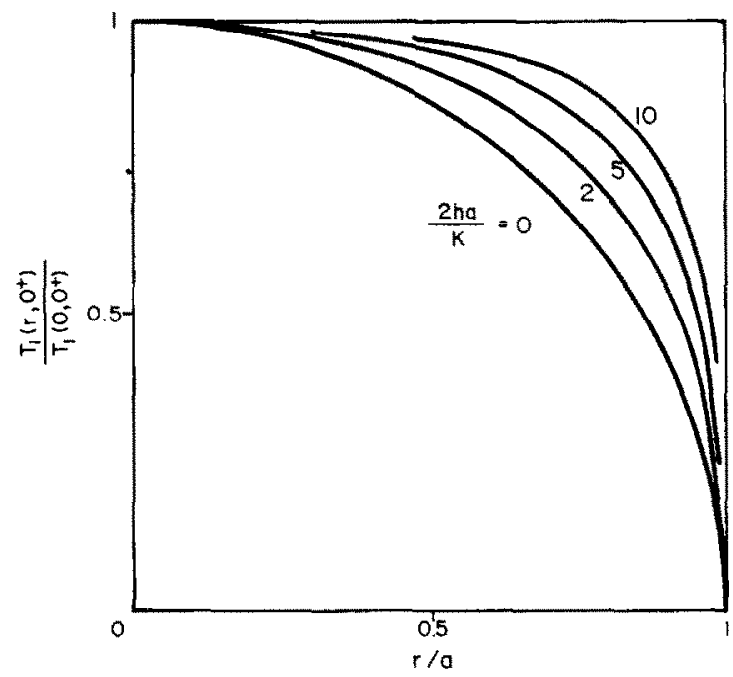

FIG. 1. Temperature profile actoss the penny-shaped crack for various values of the parameter $2 \mathrm{ha} / \mathrm{K}$.
For the extreme case in which the crack is completely non-conducting $(h=0)$ we find

$$
T_{1}\left(r, 0^{+}\right)=\frac{-2 q_{0} \sqrt{ }\left(a^{2}-r^{2}\right)}{\pi K}, \quad 0 \leqslant r \leqslant a .
$$

At the other extreme, when $2 h a / K$ is very large, the first term in equation (9) can be neglected and we obtain

$$
j(t)=\frac{q_{0} t}{\pi h \sqrt{\left(1-t^{2}\right)}}
$$

and

$$
T_{1}\left(r, 0^{+}\right)=\frac{-q_{0}}{2 h}
$$

In other words, the heat flux is scarcely disturbed by the crack, across which a small uniform temperature difference is developed.

The temperature at the centre of the crack face, $T_{1}\left(0,0^{+}\right)$ is shown in Fig. 2 as a function of $2 h a / K$, the asymptotic expression (16) being shown dotted.

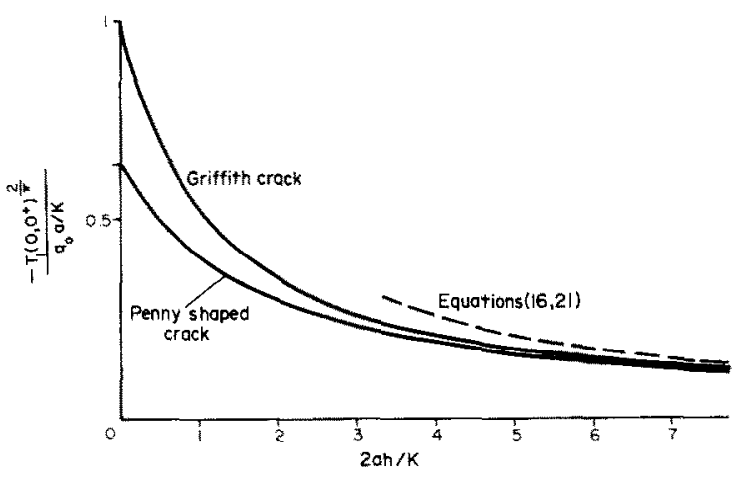

Fig. 2. Temperature at the centre of the crack face, $T_{1}\left(0,0^{+}\right)$, as a function of $2 h a / K$. The asymptotic expression (16), (21) is shown dotted.

THE GRIFFTH CRACK: $A \equiv|x|<a$

For the two-dimensional problem it is convenient to represent the crack surface temperature by the Fourier series

$$
T_{1}\left(x, 0^{+}\right)=\sum_{i=1}^{\infty} a_{i} \sin (2 i-1) \phi, \quad 0 \leqslant \phi \leqslant \pi,
$$

where $\cos \phi=x / a$. The properties of the point source and the condition (7) then enable us to develop the system of equations

$$
\frac{2 h a}{K} \sum_{j=1}^{\infty} c_{i j} a_{i}+(2 i-1) a_{i}=\frac{-q_{0} a \delta_{i 1}}{K} \text {, for } i=1,2,3 \ldots
$$

for the coefficients $a_{i}$, where

$$
c_{i j}=-\frac{2}{\pi}\left\{\frac{1}{4(i-j)^{2}-1}-\frac{1}{4(i+j-1)^{2}-1}\right\} .
$$

For a detailed derivation of this result see [2].

The form of the temperature profile across the crack is broadly similar to that obtained for the penny-shaped crack (Fig. 1) at values of $2 h a / K<10$. Limiting expressions for small and large values of $2 h a / K$-the two-dimensional equivalents of equations (14) and (16)-are

$$
\begin{aligned}
T_{1}\left(x, 0^{+}\right) & =\frac{-q_{0} \sqrt{\left(a^{2}-x^{2}\right)}}{K}, 0 \leqslant|x| \leqslant a, \frac{2 h a}{K}=0 . \\
& =\frac{-q_{0}}{2 h}, 0 \leqslant|x| \leqslant a, \frac{2 h a}{K} \gg 1 .
\end{aligned}
$$

The temperature at the mid point of the crack face. $T_{1}\left(0,0^{+}\right)$ is shown in Fig. 2 as a function of $2 h a / K$. 


\section{REFERENCES}

1. W. Karush and G. Young, Temperature rise in a hea producing solid behind a surface defect, J. Appl. Phys 23, 119 (-1193 (1952).

2. J. R. Barber, Some thermoelastic contact problems in volving frictional heating, $Q . J /$ Mech. Appl. Math. 29 1-13 (1976)

3. W. D. Collins, On the solution of some axisymmetric boundary value problems by means of integral equations --II. Further problems for a circular disc and a spherical cap, Mathematika 6, 120133 (1959). 